\title{
Experimental model of chronic osteomyelitis caused by Leishmania (L) amazonensis
}

\author{
Af Ali U.M. Costa ${ }^{a, d}$, Ana L. Abreu ${ }^{b}$, Claudia M.C. Gomes ${ }^{d}$, Ana C.R. Saldanha ${ }^{c}$, \\ A. Barral ${ }^{\text {c }}$, Jackson M.L. Costa ${ }^{\mathrm{a}, \mathrm{c}}$, Carlos E.P. Corbett ${ }^{\mathrm{d}, *}$ \\ a Nucleus of Tropical Pathology and Social Medicine, Federal University of Maranhão, São Luís, Maranhão, Brazil \\ ${ }^{\mathrm{b}}$ Laboratory of Histology, Faculty of Veterinary Medicine, State University of Maranhão, São Luís, Maranhão, Brazil \\ ${ }^{c}$ Gonçalo Moniz Research Center, FIOCRUZ/BA, Salvador, Bahia, Brazil \\ ${ }^{\mathrm{d}}$ Laboratory of Pathology of Infectious Diseases (LIM 50), Department of Pathology, Faculty of Medicine, \\ University of São Paulo, São Paulo, SP, Brazil
}

Received 3 September 2004; received in revised form 25 January 2006; accepted 16 February 2006

Available online 11 May 2006

\begin{abstract}
Experimental animal models have been used for the study of the physiopathogenesis of leishmaniasis, on some occasions with success, while in other situations such as bone alterations that accompany tegumentary leishmaniasis, especially in diffuse cutaneous form (DCL), the mechanisms are still unknown. In the present study, we determined these alterations in an animal model susceptible to Leishmania (L) amazonensis. Amastigotes of L. $(L)$ amazonensis isolated from patients with diffuse cutaneous leishmaniasis (DCL) were inoculated into the hind paws of eight BALB/c mice, macroscopic and histopathological aspects were analyzed. After 90 and 120 days of evolution, histopathological analysis demonstrated a mononuclear cell infiltrate rich in plasma cells and intense parasitism of intra- and extra-medullary macrophages, with areas of bone necrosis and discrete involvement of cartilaginous tissue. The results show that the inflammatory process developed during $L$. $(L)$ amazonensis infection might cause bone tissue destruction and secondarily affect the joints.
\end{abstract}

(C) 2006 Published by Elsevier B.V.

Keywords: Osteomyelitis; Pathology; Diffuse cutaneous leishmaniasis; Experimental model

\section{Introduction}

Although osteomyelitis is an old disease, little is known regarding its physiopathogenesis, diagnosis and treatment, and no ideal experimental animal model exists for its complete study (Norden, 1988).

\footnotetext{
* Corresponding author at: Laboratório de Imunoparasitologia (LIP) do Centro de Pesquisas Gonçalo Moniz-FIOCRUZ/BA, Rua Valdemar Falcão, 121, Brotas/Salvador-Bahia, CEP 40295-001, Brazil.

E-mail addresses: aliuthant@terra.com.br (A.A.U.M. Costa), jcosta@cpqgm.fiocruz.br (J.M.L. Costa).
}

Waldvogel and Vasey (1980), reported that the high success rates obtained for antibiotic treatment of infectious diseases contrast to those obtained for bone or joint disorders. As shown in histopathological studies, bone and joint diseases generally manifest as a granulomatous chronic inflammatory reaction and can be grouped together as being of bacterial and/or mycotic etiology, with the establishment of a diagnosis or choice of adequate treatment being difficult (Pritchard, 1975; Marrero et al., 2001). In American tegumentary leishmaniasis (ATL), some investigators have described cases of involvement of bone struc- 
tures but without explaining the mechanisms responsible for these alterations (Costa, 1953; Saldanha et al., 1995).

Mice have been widely employed as an experimental model mainly because of their physiological and genetic characteristics and easy handling (Druille et al., 2002). Infections such as Chagas disease, leprosy and tuberculosis are studied using mice of the Mus musculus lineage (Gonçalves da Costa, 1999; Druille et al., 2002). In leishmaniasis, BALB/c mice are used due to their different susceptibility to given Leishmania species, with this line apparently being more susceptible to infection with Leishmania (L) amazonensis (Perez et al., 1978; Barral et al., 1983).

Tegumentary leishmaniasis (TL) presents a rare clinical form called diffuse cutaneous leishmaniasis (DCL), described first from clinical, histopathological and parasitological aspects in Brazil (Silva, 1945) and Venezuela (Convit and Lapenta, 1946). Later, this denomination was generally used also for cases with localized lesions, which disseminated within a variable period of months to years (Bryceson, 1969). In DCL, the parasite is disseminated through the hematogenic and/or lymphatic route, and the disease evolves with the occurrence of other similar lesions in various segments of the body (Convit et al., 1972).

In bone diseases, studies on the etiological agents and their physiopathogenic mechanisms in the human organism facilitate treatment. However, there are countless unknown microorganisms causing osteomyelitis (Kahn and Pritzker, 1973; Waldvogel and Vasey, 1980; Mader et al., 1996).

Few reports are available in regarding the involvement of bone tissue in TL, and the pathogenesis of these lesions in DCL is unknown. Therefore, the aim of the present study was to reproduce infection in an animal model (BALB/c mice) using $L$. $(L)$ amazonensis strains isolated from individuals with DCL in order to better understand the histological alterations and the role of this parasite in bone lesions.

\section{Materials and methods}

\subsection{Animals}

Eight adult ( 8 weeks old) male BALB/c mice obtained from the animal house of the Laboratory of Pathology of Infectious Diseases (LIM 50), Department of Pathology, Faculty of Medicine, University of São Paulo (FMUSP), were used. The animals were housed in appropriate polyethylene cages at ambient temperature and with ample ventilation under a natural light/dark cycle, with water and food being available ad libitum. The animals passed through an 8-day adaptation period at LIM 50 before the experiment. The ethical guidelines for the practice of vivisection of animals in Brazil established by law No. 6.638 of 8 May 1979 were followed (Pimenta and Silva, 2001).

\subsection{Parasite isolation, cultivation and characterization}

Parasites were isolated by puncture aspiration of the cutaneous lesions from four patients with DCL and were cultured in tubes of NNN blood agar overlaid with a modified liver infusion tryptose (LIT) medium. Cultures were expanded for growth in modified LIT medium and kept at $25^{\circ} \mathrm{C}$. Procedures for both serodeme analysis with monoclonal antibodies and 15-enzyme electrophoresis have been previously realized (Barral et al., 1991, 1995).

\subsection{Experimental design}

The study was carried out at LIM 50, Department of Pathology, FMUSP, and laboratory of immunoparasitology (LIP), Gonçalo Moniz Research Center, FIOCRUZ/Bahia. Pairs of mice were inoculated subcutaneously into the hind foot pad with $10^{7}$ amastigotes of each strains (MHOM/BR/90/BA 338; MHOM/BR/90/BA 760; MHOM/BR/90/BA 821; MHOM/BR/90/BA 337) of Leishmania L. amazonensis and then killed after 90 days (strains 338 and 760) or 120 days (strains 821 and 337). The animals were labeled with picric acid, kept isolated during the clinical evolution of the disease, and then sacrificed.

\subsection{Surgical procedure}

The surgical procedures were always performed by the same examiner previously trained in a pilot study as proposed by Müller (2003). At the beginning of the experiment, the animals were sedated for 3-4 min in an individual chamber containing cotton soaked with sulfuric ether. After sacrifice, the animal was positioned in ventral decubitus and its limbs were fixed to the bench. The skin in the posterior region of the right hind paw was shaved disinfected with $70 \%$ alcohol and carefully dissected from the lower third of the triceps surae to the paw in a laminar flow environment. The bone tissue fragments were placed in individual containers containing $10 \%$ formalin and sent to the Laboratory of Histopathology, FMUSP, for subsequent examination. 


\section{Results}

\subsection{Macroscopic aspect}

Lymphatic involvement (hypertrophy) was observed in the limbs inoculated with the parasite. In addition, intensive necrosis with progressive edema was noted in the hind paws of the animals. Figs. 1 and 2 show the clinical following at 90 and 120 days of evolution, respectively.

\subsection{Histopathological aspect}

All animals showed destruction of soft tissues and long bone necrosis characterized by pyknotic osteocytes and empty lacunae. The presence of intense parasitism inside macrophages was observed and a mononuclear cell infiltrate rich in plasma cells with predominance of eosinophils was noted adjacent to cortical bone and in the medullary canal. In addition, there were a discrete number of osteoclasts and osteoblasts containing flattened nucleus. These aspects are illustrated in Figs. 3-5.

No limit of the invasive process was observed, i.e., whether bone tissue destruction started in the bone marrow propagating until cortical bone, or in the inverse direction. Fig. 6 characterizes this process. In some bone, fibrous tissue was detected at the distal end and in projections of trabecular bone at the proximal end. Formation of primary bone was noted in the periosteal region. Discrete cartilage involvement was observed in some animals, and secondary bacterial infection was present in adjacent soft tissues. This aspect is illustrated in Figs. 7 and 8.

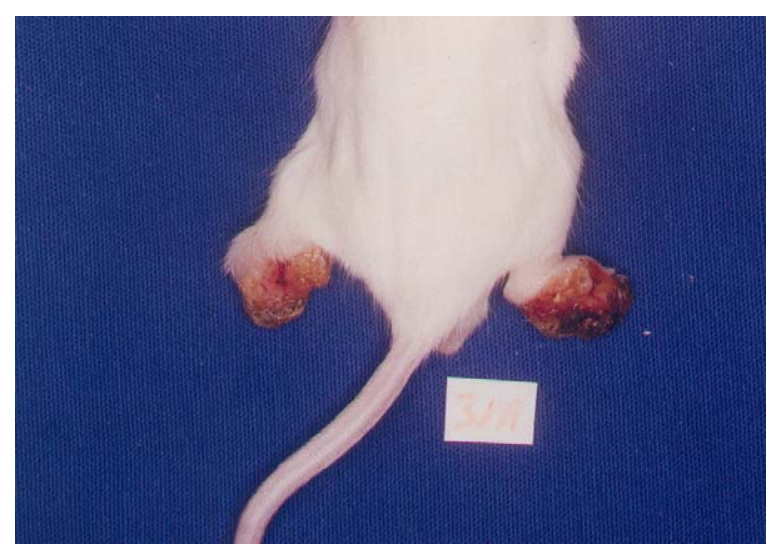

Fig. 1. Lesion in a BALB/c mouse inoculated with L. amazonensis showing histiocytomas in both paws 90 days after inoculation of the parasite.

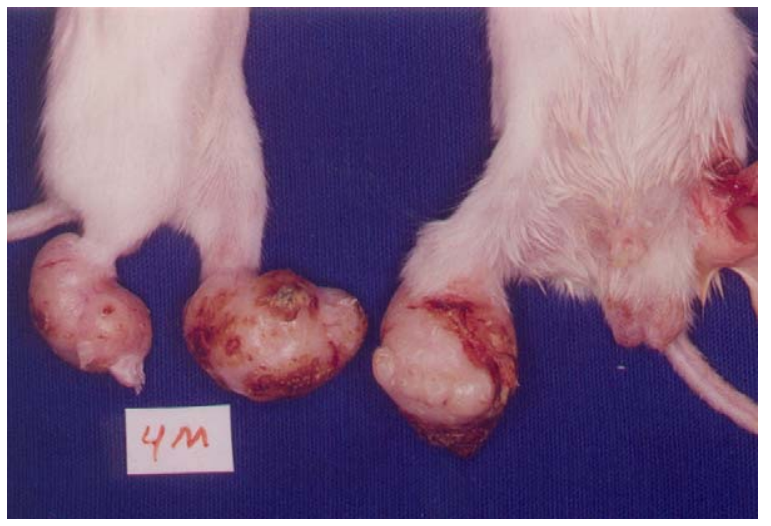

Fig. 2. Lesion in a BALB/c mouse inoculated with L. amazonensis showing, increased histiocytoma 120 days after inoculation of the parasite.

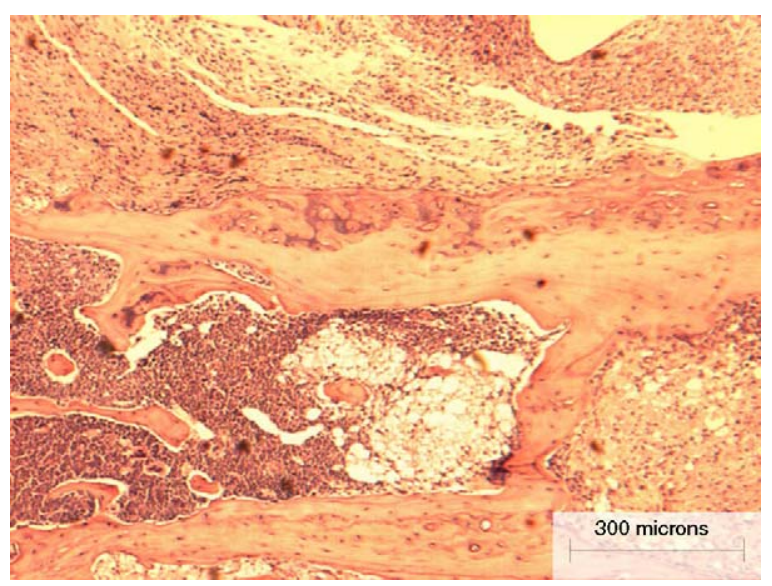

Fig. 3. Bone lesion showing bone marrow alterations, destruction of trabeculae and an inflammatory infiltrate 120 days after inoculation of the parasite.

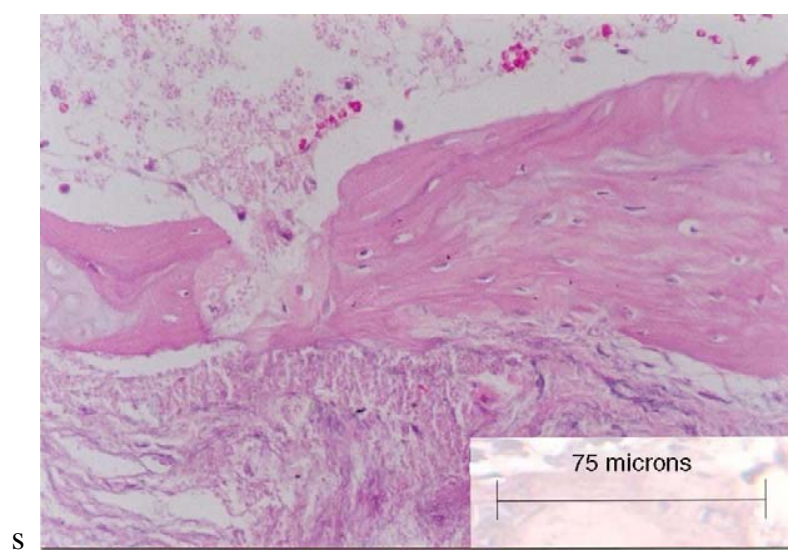

Fig. 4. Bone lesion showing cortical bone destruction accompanied by invasion of an inflammatory infiltrate 90 days after inoculation of the parasite. 


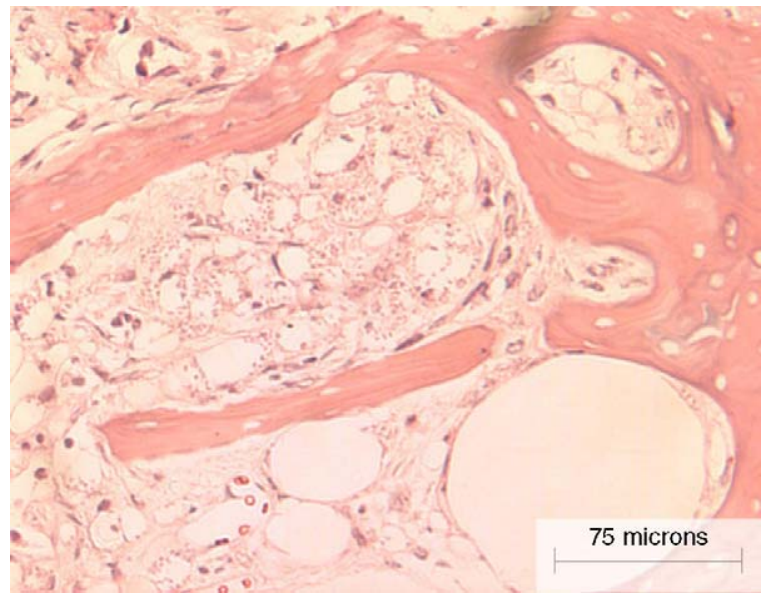

Fig. 5. Sequestrum bone and parasitized macrophages 120 days after inoculation of the parasite.

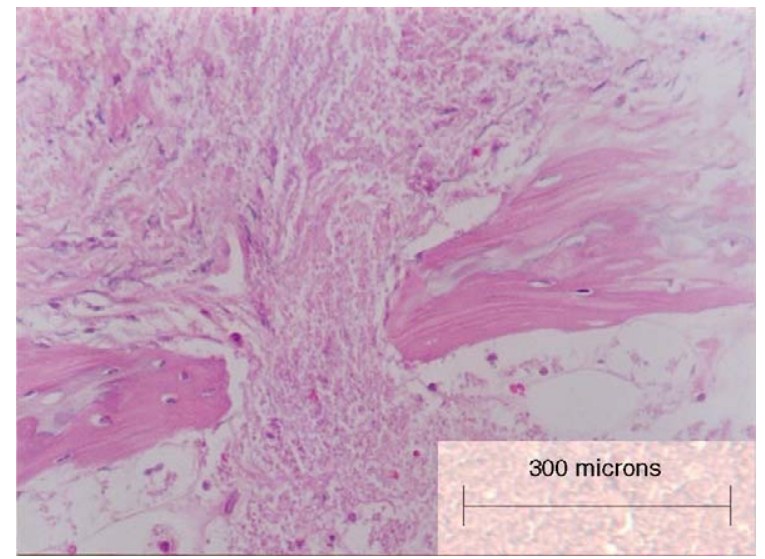

Fig. 6. Bone lesion showing cortical destruction and invasion of an inflammatory process 120 days after inoculation of the parasite.

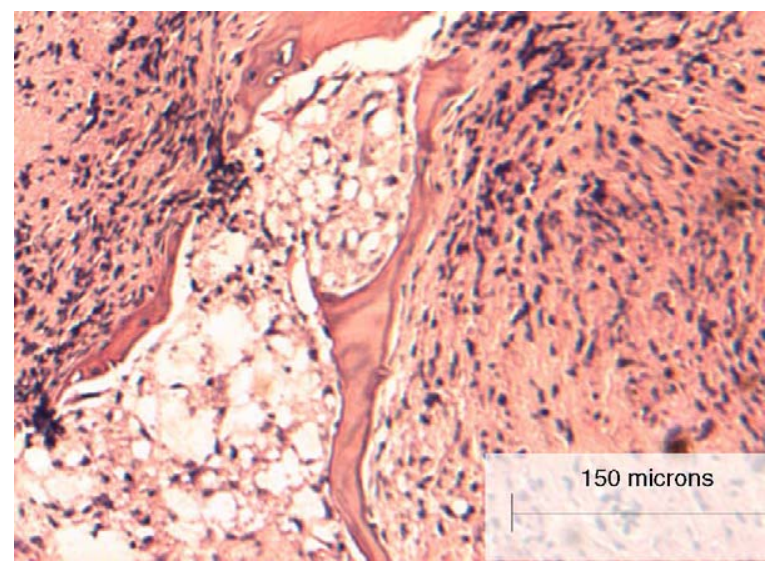

Fig. 7. Bone marrow containing parasitized macrophages with a diffuse infiltrate and destruction of trabecular bone 90 days after inoculation of the parasite.

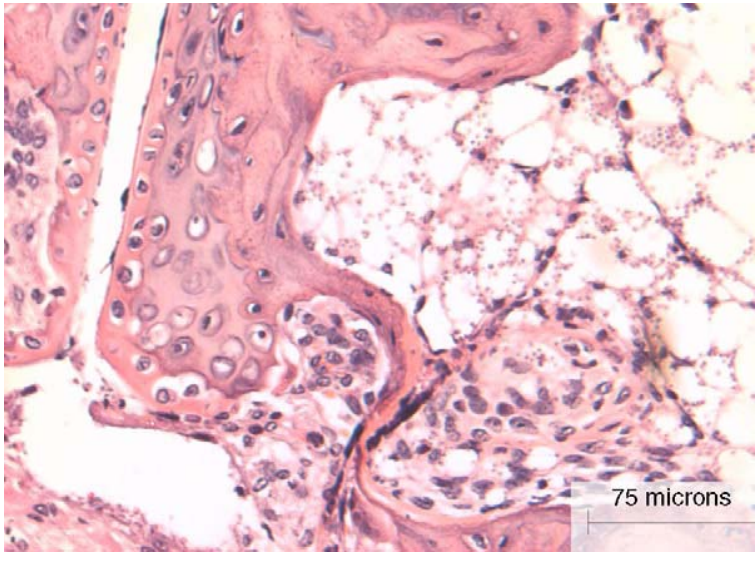

Fig. 8. Parasitized macrophages in bone marrow with extramedullary inflammatory infiltrate, 120 days after inoculation of Leishmania.

\section{Discussion}

Analysis of the history of TL shows the need for understanding the alterations that occur in bone tissue and to exclude other diseases that attack bone. However, some theories have tried to explain the pathogenesis of TL, even though few studies on this subject using laboratory assays are available (Saldanha et al., 1995).

Some investigations have reported the occurrence of bone lesions in dogs infected with $L$. $(L)$ donovani and have called attention to the natural development of manifestations of osteomyelitis associated with arthrosynovitis, suggesting that leishmaniasis should be included in the differential diagnosis of diseases that show signs of synovitis and radiographic findings of osteomyelitis in dogs from endemic areas (Turrel and Pool, 1982; Burraco et al., 1997).

In the present study, the characteristic manifestation of bone necrosis was observed in most animals, accompanied by the presence of sequestra and intense parasitism; in addition, cartilaginous tissue involvement was noted in some joints. Recently, in an experimental study, Abreu (2003), demonstrated the presence of $L$. $(L)$ amazonensis isolated from patients with the cutaneous form of TL, in various tissues such as central nervous system and bone of BALB/c and C57B/6 mice. Bone tissue involvement was detected 40 days after inoculation of the parasite into the paw, with histopathological aspects including pyknotic osteocytes, empty lacunae, and intense bone resorption in some animals, with the consequent formation of loose connective tissue. These findings were similar to those of the present study that showed massive bone tissue destruction and preservation of the articular surfaces, suggesting an inflammatory process induced by $L$. $(L)$ amazonensis in the bone marrow 
and extramedullary tissue and demonstrated by the large number of parasitized cells, with secondary involvement of cartilaginous tissue.

Although various etiological agents of osteomyelitis are known, a better understanding of the physiopathology of its subacute and chronic forms is required because of the different factors that act on bone tissue such as uncommon microorganisms, associations between these agents, and changes in vascular supply (Kahn and Pritzker, 1973; Waldvogel and Vasey, 1980).

The etiological classifications of bone infection do not cite the parasite of TL as a causative agent of osteomyelitis. In addition, few studies regarding bone involvement in TL, especially in the DCL, are available in the literature, probably because a search for Leishmania is not carried out when identifying possible responsible agents even in endemic areas, where research is only directed at bacteria, fungi and viruses. In the present study, a direct relationship between $L$. $(L)$ amazonensis and bone infection was evident, characterizing a picture of chronic osteomyelitis caused by this parasite, particularly in DCL.

\section{Acknowledgements}

We thank the Laboratory of Pathology of Infectious Diseases (LIM 50), Department of Pathology, Faculty of Medicine, University of São Paulo, for technical support and Aldina Barral (Gonçalo Moniz Research Center) for isolating the strains of Leishmania. This study was supported by CNPq.

J. Costa, has a research fellowship from Fundação de Amparo a Pesquisa da Bahia-FAPESB/Brazil and C. Corbett, and A. Barral from Conselho Nacional de Pesquisa-CNPq.

\section{References}

Abreu, A.L., 2003. Estudo da visceralização da Leishmania (L.) amazonensis no modelo murino (thesis). Rio de Janeiro: Instituto Oswaldo Cruz/FIOCRUZ, Brazil.

Barral, A., Petersen, A.A., Sacks, D.L., Neva, F.A., 1983. Late metastatic leishmaniasis in the mouse. Am. J. Trop. Med. Hyg. 35, 732-734.

Barral, A., Pedral-Sampaio, D., Grimaldi, G., Momen, H., McmahonPratt, D., Ribeiro de Jesus, A., Almeida, R., Badaró, R., BarralNetto, M., Carvalho, E.M., Johnson, W.D., 1991. Leishmaniasis in Bahia: evidence that Leishmania amazonensis produces a wide spectrum of clinical disease. Am. J. Trop. Med. Hyg. 44, 536-546.
Barral, A., Costa, J.M.L., Bittencourt, A.L., Barral-Netto, M., Carvalho, E.M., 1995. Polar and subpolar diffuse cutaneous leishmaniasis in Brazil: clinical and immunopathologic aspects. Int. J. Derm. 33, 474-479.

Bryceson, A.D.M., 1969. Diffuse cutaneous leishmaniasis in Ethiopia. I. The clinical and histological features of disease. Trans. Roy. Soc. Trop. Med. Hyg. 63, 708-737.

Burraco, P., Abate, O., Guglielmino, R., Morello, E., 1997. Osteomyelitis and arthrosynovitis associated with Leishmania donovani. Infection in a dog. J. Small Anim. Pract. 38, 29-30.

Convit, J., Lapenta, P., 1946. Sobre un caso de leishmaniasis disseminada. Rev. Pat. Clin. (Caracas) 17, 153-158.

Convit, J., Pinardi, M.E., Rondon, A.J., 1972. Diffuse cutaneous leishmaniasis: a disease due to an immunological defect of the host. Trans. Roy. Soc. Trop. Med. Hyg. 66, 609-610.

Costa, O.G., 1953. Dactilitis Leishmaniotica. Arch. Arg. Derm. 3, 149-153.

Druille, P., Hagan, P., Rook, G.A.W., 2002. The importance of models of infection in the study of disease resistance. Trends Microb. 100, S38-S46.

Gonçalves da Costa, S.C., 1999. Mouse model for Chagas disease. Does mouse represent a good model for Chagas disease? Mem. Inst. Oswaldo Cruz 94, 269-272.

Kahn, D.S., Pritzker, K.P.H., 1973. The pathophysiology of bone infection. Clin. Orthop. Rel. Res. 96, 12-19.

Mader, J.T., Ortiz, M., Calhoun, J.H., 1996. Update on the diagnosis and management of osteomyelitis. Clin. Ped. Med. Surg. 13, 701-724.

Marrero, C.M., Merino, A.J., Rodrigo, P.J., Bartolomé, A.M., Camino, F.A., Grande, S.C., 2001. Chronic recurrent multifocal osteomyelitis. Anim. Esp. Ped. 54, 181-184.

Müller, S.S., 2003. Avaliação biomecânica de sutura tendinosa com três tipos de fios cirúrgicos: estudo experimental em cães. Rev. Bras. Ortop. 38, 117-126.

Norden, C.W., 1988. Lessons learn from animal models of osteomyelitis. Rev. Inf. Dis. 10, 103-110.

Perez, H., Arredondo, B., Gonzalez, M., 1978. Comparative study of American cutaneous leishmaniasis and diffuse cutaneous leishmaniasis in two strains of inbred mice. Inf. Immun. 22, 301-307.

Pimenta, L.G., Silva, A.L., 2001. Ética e experimentação animal. Acta Cir. Bras. 16, 80-86.

Pritchard, D.J., 1975. Granulomatous infections of bones and joints. Orthop. Clin. North Am. 6, 1029-1047.

Saldanha, A.C., Malheiros, T.S., Rodrigues, C.C.R., Balby, I.T.A., Costa, J.M.L., 1995. Alterações ósseas observadas na leishmaniose cutânea difusa (LCD) no Estado do Maranhão. Rev. Soc. Bras. Med. Trop. 28, 55-56.

Silva, F., 1945. Forma raríssima de leishmaniose tegumentar. Leishmaniose dérmica não ulcerada em nódulos e extensa placas infiltradas e hiperpigmentadas. Separata do volume de Reunião Anual de Dermato-sifilografos brasileiros, Rio de Janeiro, pp. 97103.

Turrel, J.M., Pool, R.R., 1982. Bone lesions in four dogs with visceral leishmaniasis. Vet. Radiol. 23, 243-249.

Waldvogel, F.A., Vasey, H., 1980. Osteomyelitis: the past decade. N. Engl. J. Med. 303, 360-370. 